\title{
microRNA-18a, a member of the oncogenic miR-17-92 cluster, targets Dicer and suppresses cell proliferation in bladder cancer $\mathbf{T} 24$ cells
}

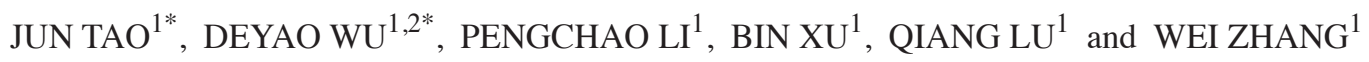 \\ ${ }^{1}$ Department of Urology, The First Affiliated Hospital of Nanjing Medical University, Nanjing, Jiangsu 210029; \\ ${ }^{2}$ Department of Urology, The Fourth Affiliated Hospital of Nantong Medical College, Yancheng, Jiangsu 224001, P.R. China
}

Received May 3, 2011; Accepted September 5, 2011

DOI: $10.3892 / \mathrm{mmr} .2011 .591$

\begin{abstract}
The miR-17-92 cluster has long been recognized as an oncogenic microRNA (miRNA) cluster and is amplified in multiple cancers. However, the individual roles of its members in carcinogenesis are largely undetermined. After transfection of miR-18a mimics, an antisense oligonucleotides inhibitor, siRNAs and a luciferase reporter plasmid, the MTT assay, colony formation assay, semi-quantitative RT-PCR, luciferase assay and Western blot analysis were conducted in bladder cancer cells. In the present study, we showed that miR-18a, a member of the miR-17-92 cluster, suppressed cell proliferation in bladder cancer T24 cells. Furthermore, ectopic expression of miR-18a in T24 cells down-regulated Dicer expression at both the mRNA and protein level, while inhibition miR-18a by antisense oligonucleotides could enhance Dicer expression in T24 cells. Two binding sites of miR-18a were found in Dicer 3' untranslated region (3' UTR). Luciferase reporter assay demonstrated that both sites could mediate expression suppression in vitro. In addition, knockdown of Dicer expression by siRNA mimicked cell growth suppression induced by miR-18a in T24 cells. These results show that miR-18a functions as a tumor suppressor by targeting Dicer in bladder cancer T24 cells and revealed a noteworthy feedback loop, which may be utilized by the miR-17-92 cluster to control miRNA output and prevent its overexpression.
\end{abstract}

\section{Introduction}

microRNAs (miRNAs) are short non-coding RNAs repressing gene expression mainly through post-transcriptional regu-

Correspondence to: Dr Qiang Lu, Department of Urology, The First Affiliated Hospital of Nanjing Medical University, 300 Guangzhou Road, Nanjing, Jiangsu 210029, P.R. China

E-mail: luqiang0405@163.com

*Contributed equally

Key words: microRNA-18a, miR-17-92 cluster, bladder cancer, Dicer, T24 cells lation by loosely binding to the mRNA $3^{\prime}$ untranslated region ( $3^{\prime}$ UTR). miRNAs are transcribed by RNA polymerase II into long primer transcripts and then sequentially spliced into mature miRNA by Drosha and Dicer endonucleases. The spectrum of miRNA targets almost covers the entire sets of genes, by which miRNAs finely regulate many cellular processes (1). Loss of miRNA biogenesis, as shown in Dicer knockout, is lethal, owing to severe defects in stem cell proliferation and differentiation $(2,3)$. It is also not surprising that miRNA may be an oncogene or a tumor suppressor gene. However, this simplified dichotomic model is in a dilemma because of the complicated and paradoxical interaction of microRNA's with their targets.

The miR-17-92 cluster is thought to play an oncogenic role in various cancer types including lung cancer (4), anaplastic thyroid cancer (5), colorectal cancer (6) as well as renal cell carcinoma (7). This cluster is located on chromosome 13q31.3, a region frequently amplified in lymphomas (8). The polycistronic miRNA cluster includes six consecutive mature miRNAs, miR-17, miR-18a, miR-19a, miR-20a, miR-19b, and miR-92a in a single primary transcript. The members contained in this cluster are frequently overexpressed in solid cancers. The sequences of these mature miRNAs are highly conserved in all vertebrates, and part of their targets is overlapped. However, each of them shows a distinct function spectrum. According to their 'seed region' similarity, these miRNAs can be classified into four categories, including miR-17/miR-20, miR-19a/miR-19b, miR-92a and miR-18a (9).

He et al showed that the miR-17-92 cluster had a direct oncogenic role by inhibiting cell apoptosis concurrent with c-myc induced lymphomas (10). Further studies demonstrated that this cluster itself was the transcriptional downstream gene of c-myc and E2Fs (11). These results gave rise to the notion that the miR-17-92 cluster may be indispensable in tumorigenesis induced by c-myc. This feedback loop was further verified by several other studies, most of which take this cluster as a whole. However, it is interesting to decipher the individual contribution of the different members to malignant transformation. Olive et al have shown that miR-19 may be a key oncogenic component of this cluster, most probably by targeting the tumor suppressor gene PTEN, while miR-18a only plays an accessory role (12). 
Table I. Primers used in this study.

\begin{tabular}{lll}
\hline Name & \multicolumn{1}{c}{ Sequence } & \multicolumn{1}{c}{ Description } \\
\hline $\begin{array}{l}\text { DICER } \\
\text { Forward }\end{array}$ & 5'-GATGGTGGTCCACGAGTCACA & $\begin{array}{l}\text { Semi-quantitative RT-PCR detection } \\
\text { of human DICER mRNA }\end{array}$ \\
Reverse & 5'-CATCAGGCAACTCTCGGGTTC & Control for semi-quantitative RT-PCR \\
Forward & 5'-GAAATCCCATCACCATCTTCCAGG & detection of human DICER mRNA \\
Reverse & 5'-GAGCCCCAGCCTTCTCCATG & For clone miR-18a binding site 1, \\
DICER clone 1 & & an enzymatic cutting site was added \\
Forward & 5'-CCCAAGCTTAATGATCTTTGGCTAAACACCC & \\
Reverse & 5'-CGAGCTCATGCAAAAGAAAAGAAGAACTGAG & For clone miR-18a binding site 2, \\
DICER clone 2 & & 5'-CCCAAGCTTGAACAGACGATAACTTTATTGGAGATTTA \\
Forward & 5'-CGAGCTCCATTGAGACCATTGTATTTTCCAC & an enzymatic cutting site was added \\
Reverse & &
\end{tabular}

We also intended to investigate the functional role of members of the miR-17-92 cluster in urinary bladder cancers. In the present study, we found that miR-18a repressed cell growth in bladder cancer T24 cells and suppressed Dicer expression by mRNA degradation as well as translational inhibition. Luciferase reporter assay demonstrated that miR-18a directly targeted Dicer 3' UTR. Furthermore, downregulation of Dicer expression by siRNA led to a marked growth supression similar to that of miR-18a in T24 cells, which indicated that sluggish cell growth induced by miR-18a might be attributed to the repression of Dicer.

\section{Materials and methods}

Cell lines and culture. The bladder cancer cell line T24 was purchased from the Chinese Academy of Science cell bank (Shanghai, China) and cultured in RPMI-1640 medium supplemented with $10 \%$ fetal bovine serum (FBS), $100 \mathrm{U} / \mathrm{ml}$ penicillin $\mathrm{G}$ and $100 \mu \mathrm{g} / \mathrm{ml}$ streptomycin. HEK293 cells were cultured in Dulbecco's modified Eagle's medium (DMEM) plus 10\% FBS, $100 \mathrm{U} / \mathrm{ml}$ penicillin $\mathrm{G}$ and $100 \mu \mathrm{g} / \mathrm{ml}$ streptomycin.

Transfection of miR-18a mimics, antisense oligonucleotides inhibitor, siRNAs and luciferase reporter plasmid. Mature miR-18a mimics, antisense oligonucleotides (ASO) inhibitor and Dicer siRNA were designed and synthesized by Genepharma (Shanghai, China). Dicer 3' UTR fragments were amplified using synthetic primers with added enzymatic cutting sites. The restriction endonuclease used for forward primers was HindIII, and that for the reverse primers was SacI (Table I). The two fragments of Dicer 3' UTR containing miR-18a binding site were 492 and 461 bp respectively. The fragments were then cloned into the pMIR-REPORT ${ }^{\mathrm{TM}}$ vector (Ambion, Austin, TX, USA). The insertion fragment was confirmed by DNA sequencing. Cell transfection and co-transfection were performed using Lipofectamine ${ }^{\mathrm{TM}} 2000$ (Invitrogen, MD, USA) according to the manufacturer's protocol.
Cell proliferation assay and clone formation assay. Cell proliferation was determined by a modified MTT method using the CellTiter $96^{\circledR}$ AQueous One solution assay (Promega, Madison, WI, USA). T24 cells (3,000 cells/well) were seeded in 96-well plates. Cell proliferation was documented every $24 \mathrm{~h}$ for 5 days according to the manufacturer's protocol. Briefly, $20 \mu \mathrm{l}$ MTS solution were added into each well and incubated at $37^{\circ} \mathrm{C}$ for $4 \mathrm{~h}$. Absorbance was measured at $490 \mathrm{~nm}$ with an ELISA reader. To evaluate the colony formation potential, $3,000 \mathrm{~T} 24$ cells were seeded in a $10 \mathrm{~cm}^{2}$ flask a day before transfection. After transfection, the cells were cultured for 10 days. Then the cells were washed with PBS solution, fixed with $4 \%$ formaldehyde for $10 \mathrm{~min}$ and dyed using crystal violet to visualize colonies. Colonies containing more than 50 cells were counted under a microscope. Ten randomly selected high power fields (HPF) were counted to obtain the mean number of colonies in each group.

Semi-quantitative reverse transcription PCR. RNA was extracted from cells using the TRIzol ${ }^{\circledR}$ reagent (Invitrogen). RNA quality control and quantification were routinely performed by spectrophotometry. A total of $500 \mathrm{ng}$ RNA were used in each reaction and GAPDH was used as an internal control. Primers for Dicer and GAPDH are shown in Table I. PCR reactions were executed under the following conditions: $95^{\circ} \mathrm{C}$ for $5 \mathrm{~min}$ followed by 30 cycles of $95^{\circ} \mathrm{C}$ for $30 \mathrm{sec}, 54^{\circ} \mathrm{C}$ for $40 \mathrm{sec}$ and $72^{\circ} \mathrm{C}$ for $45 \mathrm{sec}$. Electrophoresis on $3 \%$ agarose gels was performed to identify the amplification of the target gene. Relative expression was determined by dividing the blot density of Dicer to that of GAPDH. The experiment was repeated three times independently.

Luciferase assay. Cells were plated in a 12-well plate at $\sim 90 \%$ confluency and transfected with $0.5 \mu \mathrm{g}$ reporter plasmid, $40 \mathrm{nmol}$ miR-18a mimics or its negative control by Lipofectamine $^{\mathrm{TM}}$ 2000. Each sample was also co-transfected with $0.05 \mu \mathrm{g}$ Renilla luciferase plasmid pRL-CMV (Promega) as an internal control for transfection efficiency. The luciferase assay was performed $48 \mathrm{~h}$ after transfection using the 
A

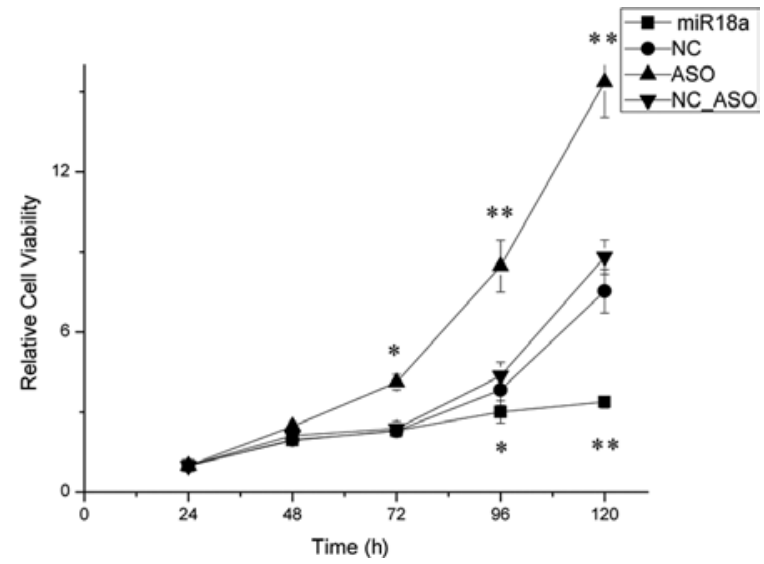

B $\mathrm{NC}$

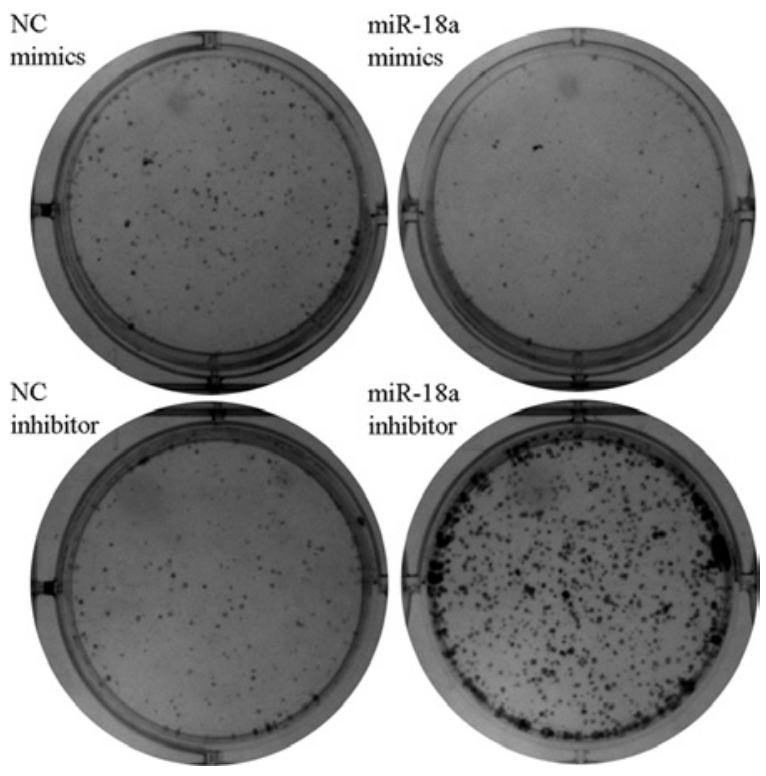

C

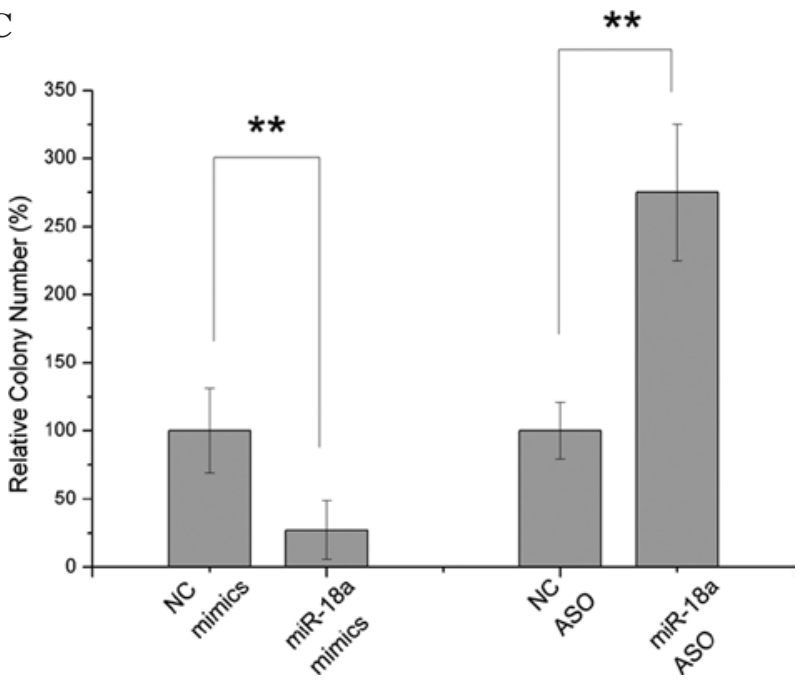

Figure 1. The effects of miR-18a on cell proliferation of bladder cancer T24 cells. (A) Cell proliferation was measured by the MTT assay. T24 cells were transfected with miR-18a mimics, antisense oligonucleotides (ASO) of miR-18a and their negative control. The MTT assay was then performed every $24 \mathrm{~h}$ for 5 days. Results are the means of three independent experiments \pm SD $\left({ }^{*} \mathrm{P}<0.05,{ }^{* *} \mathrm{P}<0.01\right)$. (B) and $(\mathrm{C})$ The effect of miR-18a on cell proliferation was evaluated by a colony formation assay. T24 cells were transfected with miR-18a mimics, ASO of miR-18a and their negative control and then seeded in 6-well plates. The number of colonies was counted on the 10th day after seeding. (B) Photos were taken at a x1 magnification. (C) Only colonies with $>50$ cells were counted. Data are presented as mean \pm SD $\left({ }^{* *} \mathrm{P}<0.01\right)$.
A
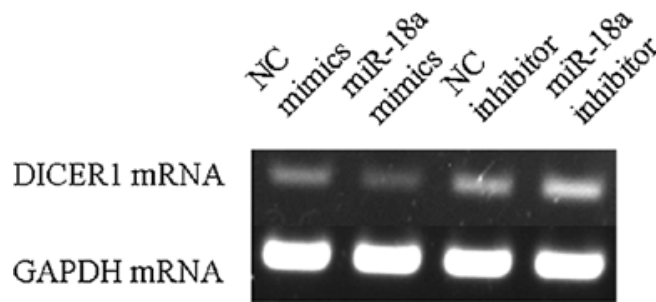

B



C

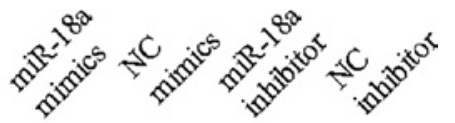

DICER

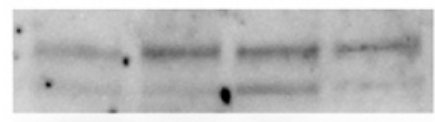

$\beta$-actin

D

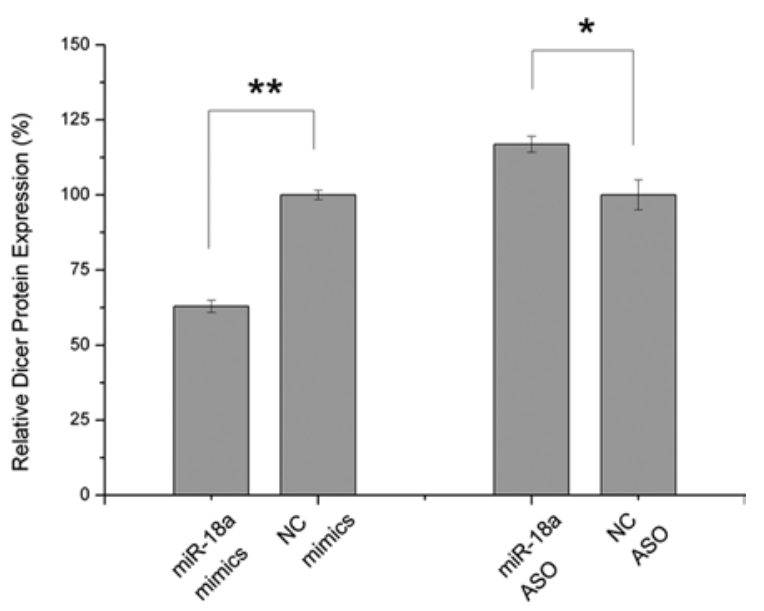

Figure 2. The effect of miR-18a on Dicer expression in T24 cells. (A) and (B) The effects of miR-18a on Dicer mRNA expression $48 \mathrm{~h}$ after transfection of miR-18a mimics, antisense oligonucleotides (ASO) of miR-18a and their negative control in T24 cells. Dicer mRNA was semi-quantitatively evaluated by RT-PCR and GAPDH was used as an internal control. Results are shown as percent changes relative to negative controls. Three independent experiments were performed and the data are presented as mean \pm SD $\left({ }^{*} \mathrm{P}<0.05,{ }^{* *} \mathrm{P}<0.01\right)$. (C) and (D) The effects of miR-18a on Dicer protein $72 \mathrm{~h}$ after transfection of miR-18a mimics, ASO of miR-18a and their negative control in T24 cells were detected by Western blotting and $\beta$-actin was used as loading control. Results are shown as percent changes relative to the negative controls and represent means of three independent experiments $\pm \mathrm{SD}$ $\left(\right.$ ( $\left.\mathrm{P}<0.05,{ }^{* *} \mathrm{P}<0.01\right)$. 
A

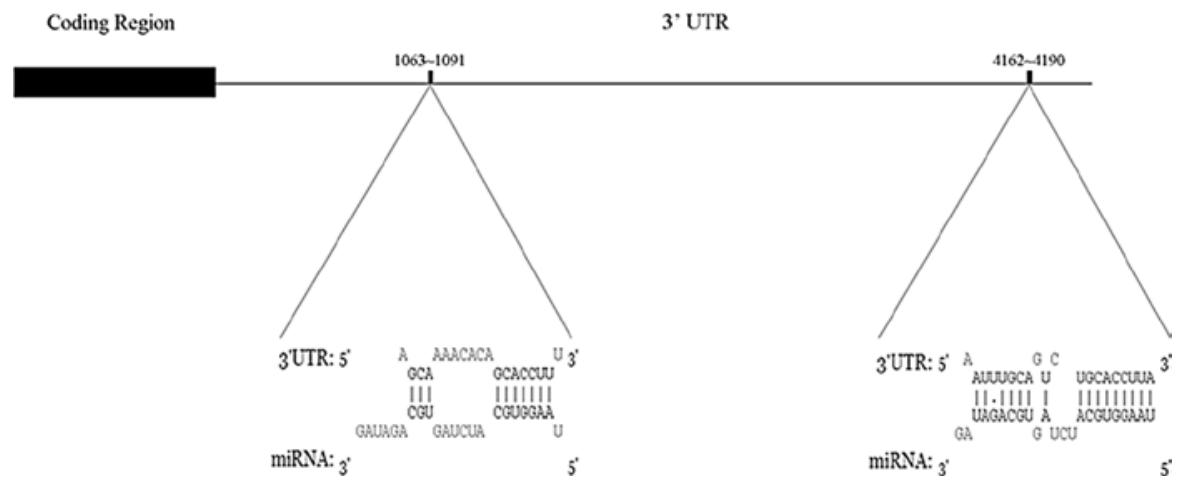

$\mathbf{B}$

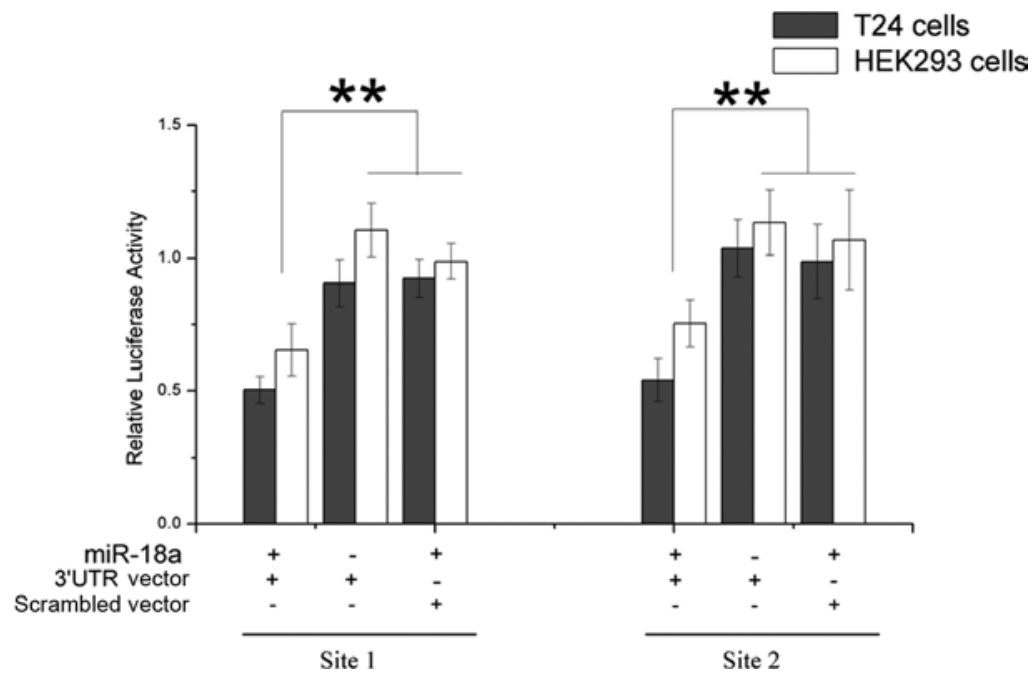

Figure 3. Dicer is a direct target of miR-18a. (A) Scheme of putative miR-18a binding sites on Dicer mRNA 3' UTR. (B) Luciferase assay verified direct binding of miR-18a with the predicted sites. Luciferase activity was significantly lowered when miR-18a was co-transfected with vectors containing its binding sites in T24 cells (dark bars) and HEK293 cells (open bars). Co-transfection with miR-18a and scrambled vector or co-transfection with negative control and vectors containing binding sites were used as controls. Three independent experiments were performed and data are presented as mean \pm SD $\left({ }^{* *} \mathrm{P}<0.01\right)$.

Dual-Luciferase ${ }^{\circledR}$ Reporter Assay system (Promega). Firefly luciferase activity was normalized to Renilla luciferase activity for each transfected well. Each assay was repeated 3 times.

Western blot analysis of Dicer protein. Antibodies of human Dicer and $\beta$-actin were purchased from Santa Cruz Biotechnology (Santa Cruz, CA, USA). Total cell lysate was prepared using RIPA buffer supplemented with phenylmethylsulfonyl fluoride (PMSF). A total of $50 \mu \mathrm{g}$ protein was loaded in each lane on $7.5 \%$ SDS-polyacrylamide gels. After electrophoresis, the protein was transferred to polyvinylidene fluoride membranes and blotted with primary and secondary antibodies. The blot was developed with ECL detection solution (Pierce, Rockford, IL, USA) and photographed using the FluorChem ${ }^{\circledR}$ imaging system (Alpha Innotech, San Leandro, CA, USA). The intensity of each spot was read and analyzed with AlphaEaseFC software. $\beta$-actin was used as a loading control.

Statistical analysis. Data are presented as mean $\pm \mathrm{SD}$, and compared using the Student' t-test in Stata v. 10 (StataCorp, College Station, TX, USA). Double-tailed $\mathrm{P}<0.05$ was considered to indicate statistically significant differences.

\section{Results}

miR-18a supresses cell proliferation in T24 cells. To investigate the influence of miR-18a on cell proliferation, we used the MTT method to survey the number of live cells after the up- or down-regulation of the miR-18a expression. Not as expected, miR-18a up-regulation did not promote cell proliferation in T24 cells. On the contrary, up-regulation of miR-18a significantly suppressed cell growth, whereas the down-regulation miR-18a by ASO obviously promoted cell growth (Fig. 1A). To confirm our observation, we performed a colony formation assay to evaluate whether miR-18a interfered with anchored cell growth in T24 cells. Consistent with our MTT results, up-regulation of miR-18a decreased colony formation by $\sim 70 \%$, whereas down-regulation of miR-18a increased colony formation $\sim 2.75$ fold (Fig. 1B and C). These results indicate that miR-18a may play a tumor suppressive role in T24 cells.

Dicer is a direct target of miR-18a. We performed a bioinformatics study to find out which targets may mediate the proliferation arrest effect of miR-18a. Interestingly, Dicer is a conserved target of miR-18a and it has been reported that inhibition Dicer expression may contribute to suppression of cell growth (13). First, we performed a semi-quantitative RT-PCR 
A

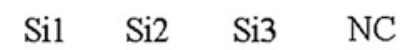

Dicer

$\beta$-actin

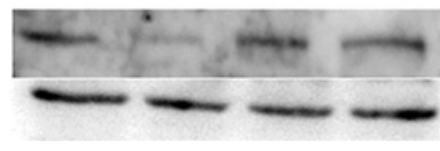

B

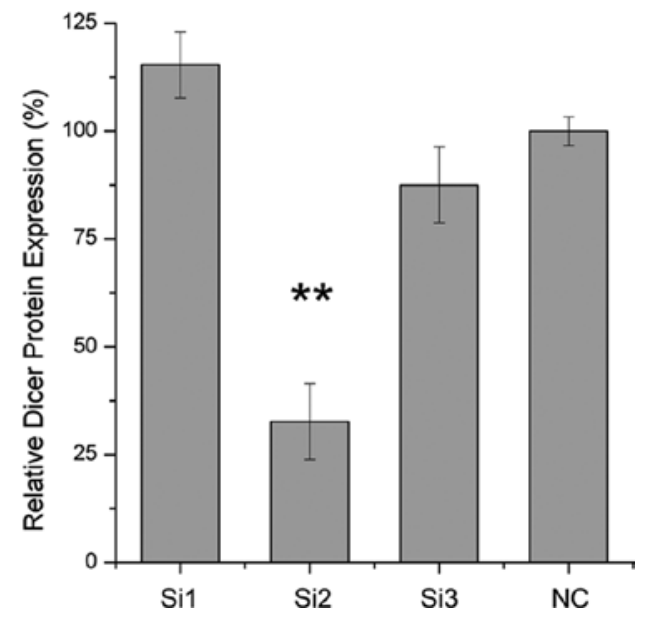

C

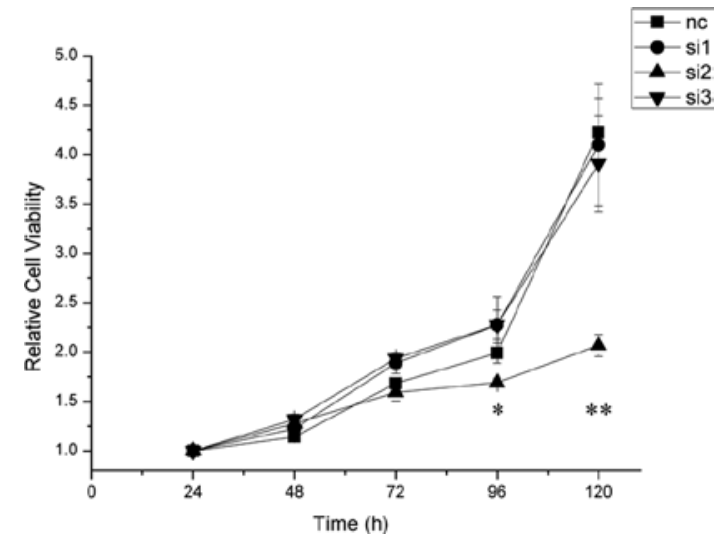

Figure 4. Effect of Dicer knockdown on cell proliferation in T24 cells. (A) and (B) Dicer knockdown by siRNA. Three distinct siRNA fragments were designed and their effects were evaluated using Western blot analysis. si2 effectively down-regulated Dicer expression as compared with the negative control or other siRNAs. (C) Cell proliferation after transfection of Dicer siRNA. T24 cells were transfected with Dicer siRNAs and negative control. The MTT assay was performed every $24 \mathrm{~h}$ for 5 days. si 2 could significantly suppress cell proliferation by knocking-down Dicer. Results are the means of three independent experiments $\pm \mathrm{SD}\left({ }^{* *} \mathrm{P}<0.01\right)$.

to evaluate how miR-18a regulated Dicer gene expression at the mRNA level. As shown in Fig. 2A and B, up-regulation of miR-18a led to $36 \%$ supression of Dicer mRNA, while down-regulation of miR-18a increased Dicer mRNA by $\sim 23 \%$. Since microRNA regulates gene expression by either mRNA degradation or translation inhibition, we performed Western blotting to detect the expression change at the protein level. As expected, miR-18a overexpression lowered Dicer protein by $\sim 40 \%$, and down-regulation of miR-18a promoted Dicer protein by $16 \%$ (Fig. 2C and D). Collectively, these findings suggest that miR-18a may target Dicer in T24 cells.

There are two conserved binding sites (S1 and S2) of miR-18a on Dicer 3' UTR predicted by bioinformatic programs, as presented schematically in Fig. 3A (14). We then cloned the 3' UTR sequences containing the S1 site
$(+1063$ from the end of coding region) and the S2 site (+4162) into the pMIR-REPORT ${ }^{\mathrm{TM}}$ vector. A luciferase assay was performed to evaluate whether these sites could directly mediate miR-18a induced suppression effect. As shown in Fig. 3B, cells co-transfected with vectors containing the S1 or $\mathrm{S} 2$ sites and miR-18a mimics showed a significant decrease in luciferase activity compared with scrambled vector plus miR-18a mimics or S1/S2 vector plus negative control. Similar results were observed when this experiment was duplicated in HEK293 cells. These results further demonstrate that Dicer might be a direct target of miR-18a.

Suppressing Dicer expression by siRNA leads to proliferation suspension in T24 cells. To evaluate whether down-regulation of Dicer results in suppression of cell proliferation, we conducted cell proliferation assays after treating T24 cells with siRNA specific to Dicer. We tested three synthetic siRNA (si1-si3) and found that si2 could suppress Dicer expression at the protein level most efficiently (Fig. 4A and B). As shown in Fig. 4C, cell proliferation was significantly supressed after transfecting si2 siRNA compared with other siRNA or the negative control. We duplicated the proliferation inhibiting effect of miR-18a by knocking down Dicer with siRNA, which indicated that miR-18a induced anti-proliferation effect may, at least partly, be attributed to its suppression of Dicer.

\section{Discussion}

The miR-17-92 cluster has long been deemed as an oncogenic miRNA cluster. However, the functions of each member are still largely undetermined. In the present study we offer the first evidence that miR-18a, one member of this cluster, might play a tumor suppressive role in bladder cancer T24 cells. We also demonstrate that this phenomenon could be due to suppression of a component of the miRNA processing machinery.

miR-18a is somewhat unique among the miR-17-92 cluster members because it shares little sequence similarity with the other members (9). We also found a different spectrum of targets for miR-18a using bioinformatics. Surprisingly, except for Dicer, many growth factors and regulatory molecules involved in tumorigenesis are also conservative targets of this miRNA, including CTGF (15), IGF1, CCND2 and HIF1A. However, studies on miR-18a are rare. Tsang et al reported that miR-18a*, a 3'-end sequence from the hairpin precursor of miR-18a, might function as a tumor suppressor by targeting the oncogene K-Ras (16). miR-18a is a minor fragment present in the cytosol with low abundance, so it is questionable to declare its significance in the physiological and pathological status. In this study, we evaluated the mature miR-18a sequence from the 5 '-end of its precursor, which is the major form of miR-18a in most cell types, and demonstrated that miR-18a suppressed cell proliferation in T24 cells by targeting Dicer. These results may reveal a novel and noteworthy mechanism which the oncogenic cluster of miR-17-92 may utilize as an endogenous brake. Furthermore, it may help us decipher the complicated relationship within this gene cluster. Because Dicer modulates miRNA biogenesis and thus controls output of mature miRNA, a negative feedback may exist to finely tune the function of the miR-17-92 cluster. 
Dicer is a ribonuclease III endonuclease with a RNA helicase motif in its amino terminus and an RNA motif in the carboxy terminus, required for siRNA and miRNA pathways to produce functional small RNA fragments (17). As a component of miRNA processing machinery, Dicer is ubiquitously expressed in almost all cell types and is crucial for miRNAmediated cellular activities. Disruption of Dicer results in embryonic lethality, demonstrating its indispensability in development (2,3). Recent studies also reveal that Dicer may have an active role in tumorigenesis and cancer progression $(18,19)$. Furthermore, as a gene with a very long 3' UTR (more than 4000 nucleotides), Dicer depends on the regulation of miRNAs, modulating the homeostasis of miRNA pathway.

Jakymiw et al have reported that let-7 miRNA could down-regulate Dicer expression, which suppresses cell proliferation in multiple oral cancer cells (18). However, Dicer down-regulation by miR-103/107 showed little effect on cell proliferation and even enhanced cell invasiveness in breast cancer cells (20). There seems to be a dose effect of Dicer in cancer cells, a severely low level of Dicer will result in cell death, while a more moderate lower level will confer a phenotype shift in cells. If this is true, it is not surprising to find that Dicer is also down-regulated in several types of cancer and is correlated with aggressive tumors $(21,22)$.

The human Dicer gene is mapped to the subtelomeric region of the short arm on chromosome 14 (14q32.13), which is vulnerable in an unstable genomic environment (23-25). Interestingly, advanced bladder cancer is characteristic of $14 \mathrm{q}$ deletion (26). Our recent findings implied that low Dicer expression occurs frequently in bladder cancers regardless of the tumor stage or grade (unpublished data). Additionally, inversion of chromosome $14 \mathrm{q}$ has been described as a typical chromosomal aberration in T24 cells, which may cause gene deletion in telomeric region or alteration of gene regulation (27). Consequently, proliferation stagnation observed in our study may be explained by further down-regulation of Dicer to a lethal threshold. However, whether or not the tumor suppressive function of miR-18a is specific to T24 cells has yet to be determined. It seems that the function of miR-18a may be dependent upon the cellular level of Dicer.

In conclusion, we have shown that miR-18a functioned as a tumor suppressor by targeting Dicer in bladder cancer T24 cells. This leaves a series of interesting questions to be answered. Further investigations are warranted to elucidate the function of miR-18a on a more extensive basis.

\section{Acknowledgements}

We are grateful to Professor Zhengdong Zhang (Department of Molecular and Genetic Toxicology, School of Public Health, Cancer Center of Nanjing Medical University, Nanjing, China) for his technical support. This study was supported by the Health Project of Jiangsu Province (XK17 20090) and the National Eleventh-Five Science and Technology Support Program of China (no. 2006BAI03B12).

\section{References}

1. Bartel DP: MicroRNAs: genomics, biogenesis, mechanism, and function. Cell 116: 281-297, 2004.
2. Bernstein E, Kim SY, Carmell MA, et al: Dicer is essential for mouse development. Nat Genet 35: 215-217, 2003.

3. Kanellopoulou C, Muljo SA, Kung AL, et al: Dicer-deficient mouse embryonic stem cells are defective in differentiation and centromeric silencing. Genes Dev 19: 489-501, 2005.

4 Hayashita Y, Osada H, Tatematsu Y, et al: A polycistronic microRNA cluster, miR-17-92, is overexpressed in human lung cancers and enhances cell proliferation. Cancer Res 65: 9628-9632, 2005.

5. Takakura S, Mitsutake N, Nakashima M, et al: Oncogenic role of miR-17-92 cluster in anaplastic thyroid cancer cells. Cancer Sci 99: 1147-1154, 2008.

6. Diosdado B, van de Wiel MA, Terhaar Sive Droste JS, et al: MiR-17-92 cluster is associated with 13q gain and c-myc expression during colorectal adenoma to adenocarcinoma progression. Br J Cancer 101: 707-714, 2009.

7. Chow TF, Mankaruos M, Scorilas A, et al: The miR-17-92 cluster is over expressed in and has an oncogenic effect on renal cell carcinoma. J Urol 183: 743-751, 2010.

8. Rinaldi A, Poretti G, Kwee I, et al: Concomitant MYC and microRNA cluster miR-17-92 (C13orf25) amplification in human mantle cell lymphoma. Leuk Lymphoma 48: 410-412, 2007.

9. van Haaften G and Agami R: Tumorigenicity of the miR-17-92 cluster distilled. Genes Dev 24: 1-4, 2010.

10. He L, Thomson JM, Hemann MT, et al: A microRNA polycistron as a potential human oncogene. Nature 435: 828-833, 2005.

11. O'Donnell KA, Wentzel EA, Zeller KI, Dang CV and Mendell JT: c-Myc-regulated microRNAs modulate E2F1 expression. Nature 435: 839-843, 2005.

12. Olive V, Bennett MJ, Walker JC, et al: miR-19 is a key oncogenic component of mir-17-92. Genes Dev 23: 2839-2849, 2009.

13. Bu Y, Lu C, Bian C, et al: Knockdown of Dicer in MCF-7 human breast carcinoma cells results in G1 arrest and increased sensitivity to cisplatin. Oncol Rep 21: 13-17, 2009.

14. Maragkakis M, Reczko M, Simossis VA, et al: DIANA-microT web server: elucidating microRNA functions through target prediction. Nucleic Acids Res 37: W273-W276, 2009.

15. Ohgawara T, Kubota S, Kawaki H, et al: Regulation of chondrocytic phenotype by micro RNA $18 \mathrm{a}$ : involvement of $\mathrm{Ccn} 2 / \mathrm{Ctgf}$ as a major target gene. FEBS Lett 583: 1006-1010, 2009.

16. Tsang WP and Kwok TT: The miR-18a* microRNA functions as a potential tumor suppressor by targeting on K-Ras. Carcinogenesis 30: 953-959, 2009.

17. Lee Y, Ahn C, Han J, et al: The nuclear RNase III Drosha initiates microRNA processing. Nature 425: 415-419, 2003.

18. Jakymiw A, Patel RS, Deming N, et al: Overexpression of dicer as a result of reduced let-7 MicroRNA levels contributes to increased cell proliferation of oral cancer cells. Genes Chromosomes Cancer 49: 549-559, 2010.

19. Chiosea S, Jelezcova E, Chandran U, et al: Overexpression of Dicer in precursor lesions of lung adenocarcinoma. Cancer Res 67: 2345-2350, 2007.

20. Martello G, Rosato A, Ferrari F, et al: A MicroRNA targeting dicer for metastasis control. Cell 141: 1195-1207, 2010.

21. Pampalakis G, Diamandis EP, Katsaros D and Sotiropoulou G: Down-regulation of dicer expression in ovarian cancer tissues. Clin Biochem 43: 324-327, 2010.

22. Wu JF, Shen W, Liu NZ, et al: Down-regulation of Dicer in hepatocellular carcinoma. Med Oncol 28: 804-809, 2010.

23. Pehlivan D, Gunduz E, Gunduz M, et al: Loss of heterozygosity at chromosome $14 \mathrm{q}$ is associated with poor prognosis in head and neck squamous cell carcinomas. J Cancer Res Clin Oncol 134: 1267-1276, 2008.

24. Felsberg J, Yan PS, Huang TH, et al: DNA methylation and allelic losses on chromosome arm 14q in oligodendroglial tumours. Neuropathol Appl Neurobiol 32: 517-524, 2006.

25. Al-Mulla F, AlFadhli S, Al-Hakim AH, Going JJ and Bitar MS: Metastatic recurrence of early-stage colorectal cancer is linked to loss of heterozygosity on chromosomes 4 and 14q. J Clin Pathol 59: 624-630, 2006.

26. Chang WY, Cairns P, Schoenberg MP, Polascik TJ and Sidransky D: Novel suppressor loci on chromosome $14 \mathrm{q}$ in primary bladder cancer. Cancer Res 55: 3246-3249, 1995.

27. Hastings RJ and Franks LM: Chromosome pattern, growth in agar and tumorigenicity in nude mice of four human bladder carcinoma cell lines. Int J Cancer 27: 15-21, 1981. 